\title{
Faster Algorithms for Computing Maximal Multirepeats in Multiple Sequences
}

\author{
Costas S. Iliopoulos* \\ Algorithm Design Group, Department of Computer Science, King's College London \\ The Strand, London WC2R 2LS, England, csi@dcs.kcl.ac.uk \\ Digital Ecosystems \& Business Intelligence Institute, Curtin University \\ GPO Box U1987, Perth WA 6845, Australia
}

W. F. Smyth ${ }^{\dagger *}$

Algorithms Research Group, Department of Computing \& Software, McMaster University

Hamilton, Ontario, Canada L8S 4K1, smyth@mcmaster.ca

Digital Ecosystems \& Business Intelligence Institute, Curtin University

GPO Box U1987, Perth WA 6845, Australia

Munina Yusufu ${ }^{\dagger *}$

Algorithms Research Group, Department of Computing \& Software, McMaster University

Hamilton, Ontario, Canada L8S 4K1, yusufum@mcmaster.ca

Digital Ecosystems \& Business Intelligence Institute, Curtin University

GPO Box U1987, Perth WA 6845, Australia

\begin{abstract}
A repeat in a string is a substring that occurs more than once. A repeat is extendible if every occurrence of the repeat has an identical letter either on the left or on the right; otherwise, it is maximal. A multirepeat is a repeat that occurs at least $m_{\min }$ times $\left(m_{\min } \geq 2\right)$ in each of at least $q \geq 1$ strings in a given set of strings. In this paper, we describe a family of efficient algorithms based on suffix arrays to compute maximal multirepeats under various constraints. Our algorithms are faster, more flexible and much more space-efficient than algorithms recently proposed for this
\end{abstract}

Address for correspondence: Munina Yusufu, Algorithms Research Group, Department of Computing \& Software, McMaster University, Hamilton, Ontario, Canada L8S 4K1, yusufum@mcmaster.ca

${ }^{*}$ The author wish to acknowledge the contribution of anonymous referees, whose suggestions materially improved this paper.

${ }^{\dagger}$ The work of the author was supported in part by the Natural Sciences \& Engineering Research Council of Canada. 
problem. The results extend recent work by two of the authors computing all maximal repeats in a single string.

Keywords: maximal multirepeats, repeats, gaps, biological sequences, suffix arrays

\section{Introduction}

In this paper, we propose efficient algorithms for finding the maximal multirepeats in a set of strings under various constraints. The problem of finding common regularities among a set of strings is very important [4]. In biological sequences (DNA, RNA, or protein) the problem of locating repeats in a set of strings (multirepeats) arises in many contexts, such as database searching and sequence alignment [1]. It is also important in data mining [8,3].

A repeat in a string is a substring that occurs more than once. The distance (number of intermediate letters) between the occurrences of the same substring is called a gap. A repeat is left-maximal, if not all occurrences have the same letter on the left, right-maximal, if not all occurrences have the same letter on the right - thus maximal if both left- and right-maximal. Reporting only maximal repeats avoids redundant reporting of repeats that are embedded in other repeats.

In [10], several fast algorithms for computing different kinds of maximal repeats under some restrictions were proposed, but only for a single string. To compute repeats in a set of strings (multiple repeats), there exists only one algorithm [1]. This algorithm is not space-efficient since it uses suffix trees, one for each string in the set plus a "generalized" suffix tree for all of them. Thus it is not easy to implement. In addition, it has high time complexity. If gaps are unrestricted, the algorithm of [1] requires $O\left(\sigma N^{2} n+\alpha\right)$ time; if gaps are required to fall in a range of length $c$, it requires $O\left(\left(c^{2}+\sigma^{2}\right) m N^{2} n \log (N n)+\alpha\right)$ time. Here $\sigma$ is the alphabet size, $N$ the number of strings, $n$ the average length of the $N$ strings, $m$ the multiplicity (number of occurrences) of the multirepeat, and $\alpha$ the total number of occurrences of all reported repeats. While $n$ may be quite large (millions), in applications $N$ is generally a small integer (at most two digits). Similarly, we may suppose that the number $R$ of reported repeats is $o(n)$. Further, in keeping with the application, we suppose throughout that alphabet size $\sigma \leq 256$, so that an individual letter requires at most one byte for storage.

Here we extend previous work [10] to the problems considered in [1], proposing algorithms that are more time-efficient, as well as being easier to implement and using much less space. We describe algorithms to find complete maximal multirepeats that occur at least $m_{\min }$ times in each of at least $q$ strings in a given set $S$ of $N$ strings, first with no restriction on gap length, then with bounded gaps. For the first problem, we propose two algorithms with worst-case time complexities $O\left(N n+\alpha \log _{2} N\right)$ and $O(N n+\alpha)$ that use $9 N n$ and $10 N n$ bytes of space, respectively. For the second problem, we describe an algorithm with worst-case time complexity $O(R N n)$ that requires approximately $10 N n$ bytes. Note that all times are independent of alphabet size. Extending the algorithms of [1], our three algorithms output only repeats whose occurrences are substrings of length at least $p_{\min }$ (user-specified), thus eliminating trivial outputs.

The remainder of the paper is organized as follows. In Section 2, we give definitions and formulate the problems. In Section 3, we give details of the three algorithms noted above. Finally, in Section 4 we give conclusions and thoughts on further research. 


\section{Preliminaries}

Basic string terminology in this paper follows [12].

\subsection{Repeats \& Data Structures}

A repeat in $x$ is a tuple $M_{x, u}=\left(p ; i_{1}, i_{2}, \ldots, i_{m}\right)$, where $m \geq 2,1 \leq i_{1}<i_{2}<\ldots<i_{m} \leq n$, and $u=x\left[i_{1} . . i_{1}+p-1\right]=x\left[i_{2} . . i_{2}+p-1\right]=\ldots=x\left[i_{m} . . i_{m}+p-1\right]$. We call $u$ the generator, $p$ the period and $m$ the multiplicity of $M_{x, u}$. If $u$ occurs at two positions $i$ and $j$ in $x$, then the distance $g=|i-j|-p$ is called a gap. Note that $g$ may be negative (overlapping occurrences) or zero (tandem occurrences).

Our second problem considers restrictions on the gaps as follows: if for $i \in 1 . . \mu-1$, where $\mu=m_{\min }$, $g_{i}$ is the gap between the $i$ th and $(i+1)$ th occurrences of $u$, then we require $d_{\min _{i}} \leq g_{i} \leq d_{\text {max }_{i}}$, lower and upper bounds on $g_{i}$. Collectively, these restrictions are represented by a $(\mu-1)$-tuple

$$
d=\left(\left(d_{\min _{1}}, d_{\max _{1}}\right),\left(d_{\min _{2}}, d_{\max _{2}}\right), \ldots,\left(d_{\min _{\mu-1}}, d_{\max _{\mu-1}}\right)\right) .
$$

As remarked above, our PSY1 algorithm [10] outputs maximal repeats of period $p \geq p_{\min }$. For this, certain well-known data structures are required.

Given a string $s=s[1 . . \ell]$ of length $\ell$, the array $s a=s a[1 . . \ell]$ is a suffix array of $s$ iff its entries are a permutation of $1 . . \ell$ such that for $j \in 1 . . \ell, s a[j]=i$ whenever suffix $s[i . . \ell]$ is the $j^{\text {th }}$ in lexicographical order among all the suffixes of $s$. For brevity, we sometimes refer to $s=s[i . . \ell]$ simply as suffix $i$. Often the suffix array is used in combination with the longest common prefix (lcp) array which gives the length of the longest common prefix between consecutive suffixes of $s a$; that is, $l c p[j]$ is the length of the longest common prefix of $s[s a[j] . . n]$ and $s[s a[j-1] . . n]$. Also required is the Burrows-Wheeler transform [2], an array $b w t=b w t[1 . . \ell]$, most simply defined as follows: for $s a[j]>1, b w t[j]=s[s a[j]-1]$, while for $j$ such that $s a[j]=1, b w t[j]=\$$, a sentinel letter less than any other letter in the alphabet.

\subsection{Formulation of Problems}

We define two problems:

Unconstrained Multirepeats (abbreviated MultiRep): Given a set $S=\left\{s_{1}, s_{2}, \ldots, s_{N}\right\}$ of strings, where each string $s_{k}, 1 \leq k \leq N$, has length $n$ (if the lengths of the strings vary, $n$ represents their average length), and a tuple of positive integers $D=\left(p_{\min }, q, m_{\min }\right)$, where $p_{\min } \geq 1, q \in 1 . . N$, $m_{\min } \geq 2$, we output all maximal multirepeats of period at least $p_{\min }$ that occur at least $m_{\min }$ times in each of at least $q$ strings of $S$. Following [1], we call $q$ the quorum and $m_{\min }$ the minimum multiplicity.

Example 1: Given a set of three strings $S=\left\{s_{1}, s_{2}, s_{3}\right\}$, with $D=(3,2,2)$, we find a maximal repeat ACG of length $p_{\min }=3$ that occurs at least $m_{\min }=2$ times in all $3 \geq q=2$ of the strings. Thus the repeat would be output. However, for $D=(3,3,3), s_{3}$ would not satisfy $m \geq 3$ and so only $2<q=3$ of the strings would have the minimum number of occurrences; in this case no output would occur.

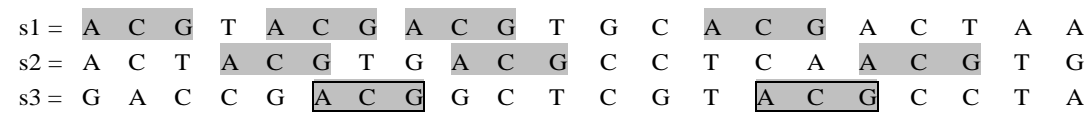


Multirepeats with Constrained Gaps (abbreviated MultiRepG): In addition to $S$ and $D$, we are given a tuple of gap constraints (1). We compute all the repeats that satisfy (1) at least $q$ times as well as the constraints $D$. More precisely, in each individual string $s_{k} \in S$ that contains $m \geq m_{\text {min }}$ occurrences of the repeating substring, we look for a sequence of $\mu=m_{\min }$ consecutive occurrences that satisfies (1); if such a sequence exists in at least $q$ strings, we output all $m$ occurrences in every $s_{k}$ for which (1) is satisfied.

Example 2: Given the same set $S$ and $D=(3,2,2)$ as in Example 1, we introduce the constraint $\left(d_{\text {min }_{i}}, d_{\text {max }_{i}}\right)=(0,5)$ for every $i \in 1 . . \mu-1$. Because the gap between $s_{3}[6 . .8]$ and $s_{3}[15 . .17]$ exceeds 5 , ACG does not satisfy the gap constraints in $s_{3}$, but continues to do so in $s_{1}$ and $s_{2}$, thus at least $q=2$ times. Thus occurrences of ACG only in $s_{1}$ and $s_{2}$ are output.

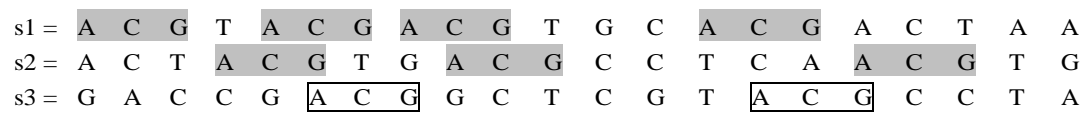

\section{Description of the Algorithms}

The overall strategy for both problems MultiRep and MultiRepG is the same:

* form a single string $s$ from the given set $S$ of $N$ strings;

* in a preprocessing phase, compute the suffix array $s a$, the longest common prefix array lcp and the Burrows-Wheeler transform bwt for $s$;

* use Algorithm PSY1 [10] to compute all maximal repeats of period $p \geq p_{\min }$ in $s$;

* output the repeats that satisfy $D$ (MultiRep) or both $D$ and $d$ (MultiRepG).

\subsection{No Constraints on Gaps}

\subsubsection{Algorithm MultiRep-1}

\begin{tabular}{|c|c|c|c|c|c|c|c|}
\hline $\mathrm{n}_{1}$ & $\mathrm{n}_{2}$ & & $\mathrm{n}_{3}$ & & ......... & $\mathrm{n}_{\mathrm{N}}$ & \\
\hline$\square$ & $\$_{1} \quad \mathrm{~s}_{2}$ & $\$ 2$ & $\mathrm{~s}_{3}$ & $\$_{3}$ & $\ldots . .$. & $\mathrm{s}_{\mathrm{N}}$ & $\$$ \\
\hline
\end{tabular}

Figure 1. Form a new string using end-of-string sentinels

From the set $S=\left\{s_{1}, s_{2}, \ldots, s_{N}\right\}$ of strings, form $s=s_{1} \$_{1} s_{2} \$_{2} s_{3} \$_{3} \ldots \$_{N-1} s_{N} \$$, as shown in Figure 1, where the end-of-string sentinels $\$_{j}, 1 \leq j \leq N-1$, and $\$$ are distinct symbols less in lexicographic order than any of the letters in the $s_{k}, 1 \leq k \leq N$, and that moreover satisfy $\$<\$_{1}<$ $\$_{2}<\ldots<\$_{N-1}$. Let $s_{k}=s_{k}\left[1 . . n_{k}\right]$. 
The preprocessing computes the $s a, l c p$ and $b w t$ arrays for $s$ using standard algorithms as described in [10]: in these algorithms the $\$_{j}$ are treated as normal letters, while $\$$ just marks the end of $s$ and is not included in calculations.

Example 3: Given $S=\left\{s_{1}, s_{2}, s_{3}\right\}$, where $s_{1}=$ AAGTCAG, $s_{2}=$ AGAG, $s_{3}=$ CAGTAGC, we form $s=s_{1} \$_{1} s_{2} \$_{2} s_{3} \$$ and preprocess.

\begin{tabular}{|c|c|c|c|c|c|c|c|c|c|c|c|c|c|c|c|c|c|c|c|c|c|}
\hline & 1 & 2 & 3 & 4 & 5 & 6 & 7 & 8 & 9 & 10 & 11 & 12 & 13 & 14 & 15 & 16 & 17 & 18 & 19 & 20 & 21 \\
\hline $\mathrm{s}$ & A & A & $\mathrm{G}$ & $\mathrm{T}$ & C & A & G & $\$_{1}$ & A & $\mathrm{G}$ & A & $\mathrm{G}$ & $\$_{2}$ & C & A & G & $\mathrm{T}$ & A & G & $\mathrm{C}$ & $\$$ \\
\hline sa & 8 & 13 & 1 & 6 & 11 & 9 & 18 & 15 & 2 & 20 & 5 & 14 & 7 & 12 & 10 & 19 & 16 & 3 & 17 & 4 & \\
\hline lcp & -1 & 0 & 0 & 1 & 2 & 2 & 2 & 2 & 3 & 0 & 1 & 3 & 0 & 1 & 1 & 1 & 1 & 2 & 0 & 1 & -1 \\
\hline bwt & G & G & $\$$ & $\mathrm{C}$ & $\mathrm{G}$ & $\$_{1}$ & $\mathrm{~T}$ & C & A & G & $\mathrm{T}$ & $\$_{2}$ & A & A & A & A & A & A & G & G & $\$$ \\
\hline
\end{tabular}

PSY1 makes use of the preprocessed arrays to compute maximal repeats, each one a triple $(p ; i, j)$ specifying a period $p \geq p_{\min }$ and a range $i . . j$ in $s a$ such that for every $h \in i . . j$, suffix $s a[h]$ has an identical prefix of length $p$, while suffixes $s a[i-1]$ and $s a[j+1]$ (if they exist) do not. If we are given $p_{\min }=2$ in Example 3, PSY1 would output only one maximal repeat for $\boldsymbol{u}=A G$ in the form $(p ; i, j)=(2 ; 4,9)$ with period $p=2$, where the range $4 . .9$ identifies $s a[4]=6, s a[5]=11, s a[6]=$ $9, s a[7]=18, s a[8]=15, s a[9]=2$. Thus the maximal repeat occurs in positions $6,11,9,18,15,2$ of $s$ as shown by the shading in Example 3.

Given an output $(p ; i, j)$ from PSY1, we need to determine if the conditions specified by the tuple $D$ are satisfied. Our first task is to use the suffix array $s a$ to convert this output into the form $M=$ $(p ; s a[i], s a[i+1], \ldots, s a[j])$ keyed to positions in $s$ rather than $s a$ : over all repeats found by PSY1, this will require $O(\alpha)$ time. We then make use of two arrays, divpts and count. Array divpts specifies the starting points of each substring $s_{k}$ of $s$ - this permits a binary search to be done to determine in which substring $s_{k}$ the current repeating substring is located. More precisely:

$$
\operatorname{divpts}[1 . . N+1]=\left[1, n_{1}+2, n_{1}+n_{2}+3, \ldots, \sum_{k=1}^{N-1}\left(n_{k}+1\right)+1, \sum_{k=1}^{N}\left(n_{k}+1\right)+1\right] .
$$

The array count $=\operatorname{count}[1 . . N]$ just maintains a count of the number of repeating substrings that have so far been found to lie within each of the $N$ strings $s_{k}$.

Using these arrays, it is straightforward to determine in time $O((j-i) \log N)$ whether the repeat $(p ; i, j)$ occurs at least $m_{\min }$ times in each of at least $q$ substrings of $s$, as shown in Figure 2. Note that if $j-i+1<m_{\min } q$, this condition cannot be satisfied and so no tests are required. The function BinarySearch called in MultiRep-1 (see Figure 2) returns the index $k$ indicating that position $s a[h]$ in $s$ occurs in substring $s_{k}$.

In Example 3, divpts will be $[1,9,14,22]$ and the output repeat will be $(2 ; 6,11,9,18,15,2)$. After binary search we find that count $[1]=2$ (positions 6 and 2), $\operatorname{count}[2]=2(11$ and 9$)$, and $\operatorname{count}[3]=2$ (18 and 15): the repeat occurs at least twice in each of the three substrings. Thus for $m_{\min }=2, q=3$, the repeat satisfies the constraints specified by $D$.

Now we analyze the time and space complexity of the algorithm. For construction of $s a$ there are algorithms linear in string length $\ell[7,5]$, though in practice algorithms with worst-case $O\left(\ell^{2} \log \ell\right)$ time requirement are several times faster [11]. To compute $l c p$ from $s a$ there are two linear-time algorithms 


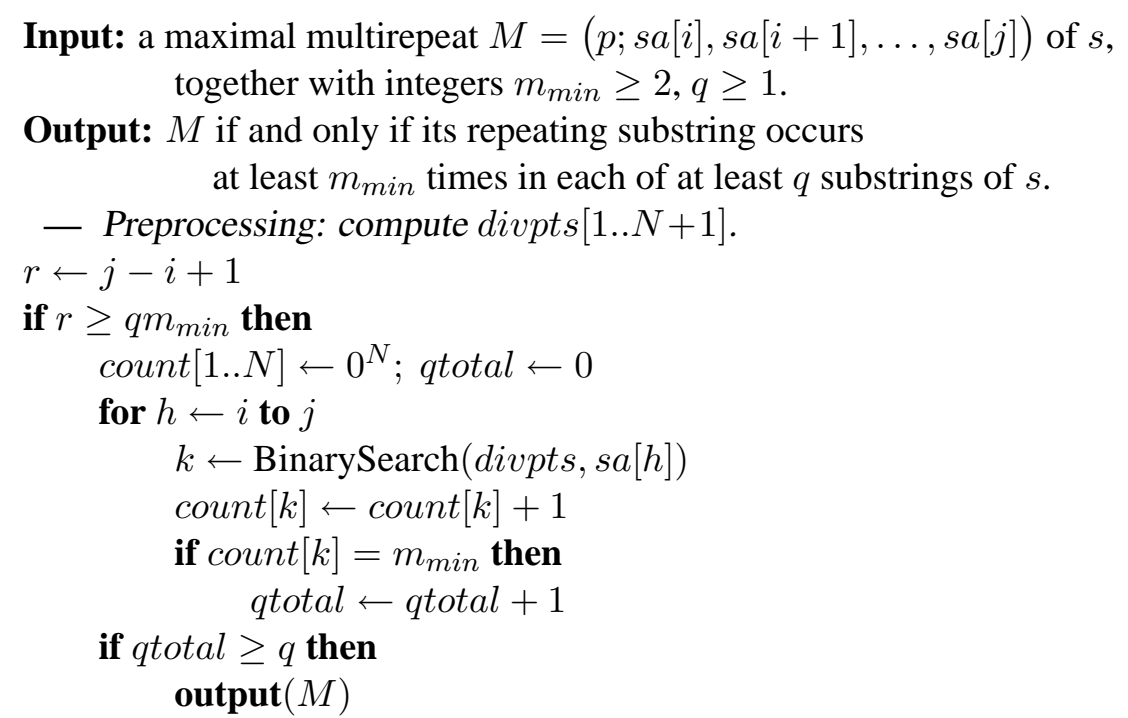

Figure 2. Algorithm MultiRep-1: Check Multiplicity \& Quorum

[6, 9], and the easy calculation of bwt from $s a$ is also linear. Given $l c p$ and $b w t$, PSY1 executes in linear time [10]. In our case $\ell=N(n+1)$, and so all the repeats $(p ; i, j)$ in $s$ can be computed in time $O(N n)$. For each of $O(R)$ repeats, the array count must be cleared at a cost of $O(N)$ time. In addition, for each of at most $\alpha$ occurrences of repeating substrings in $s$, the time required is at most $O\left(\log _{2} N\right)$ for the binary search. Thus to compute all the repeats satisfying constraint $D$, the worst-case time complexity of the algorithm shown in Figure 2 is $O\left(N n+R N+\alpha \log _{2} N\right)$.

However, the asymptotic time complexity of MultiRep-1, though not perhaps the expected running time in practice, can be slightly reduced, as we now explain. Instead of performing count $\leftarrow 0^{N}$ as part of the algorithm, execute it only once as preprocessing over all invocations of MultiRep-1. Introduce into MultiRep-1 a list $L$, initially empty, to which each value $k$ computed by BinarySearch is added; then at the end of MultiRep-1 introduce a new loop that removes from $L$ each entry $k$ and performs count $[k] \leftarrow 0$. The resulting algorithm executes in time $O\left(N n+\alpha \log _{2} N\right)$, independent of $R$.

Preprocessing for a string of length $\ell$ requires as few as $5 \ell$ bytes for $s a$ [11], $9 \ell$ for $l c p$ [9] and $6 \ell$ for $b w t$, thus at most $9 N(n+1)$ bytes for $\ell=N(n+1)$. PSY1 itself requires only $5 \ell$ bytes for its execution [10], plus a further $4 \ell$ for storage of $s a$ (since each range $i, j$ in $s a$ needs to be converted into a sequence $s a[i], s a[i+1], \ldots, s a[j]$ in $s)$. Since divpts and count are arrays $1 . . N$ of integer, their total space requirement is $8 N$ bytes, and so the total is $N(9 n+17)$ bytes, in simple terms $9 N n$.

The algorithm shown in Figure 2 outputs all of the repeats $M$. It may instead be required to output only those positions in $M$ that occur in the $s_{k}$ for which $m \geq m_{\min }$. One way to accomplish this is to introduce a Boolean array mok $=\operatorname{mok}[1 . . N]$ (similar in its role to the array gapsok described below for MultiRepG) - mok records for each $k \in 1 . . N$ whether or not $s_{k}$ contains at least $m_{\min }$ occurrences of $M$. Then a straightforward processing of $M$, again using BinarySearch, produces the required output, using the same asymptotic time and space. 


\subsubsection{Algorithm MultiRep-2}

We briefly describe a strategy to avoid the binary search of MultiRep-1, at a cost of an additional $N(n+1)$ bytes of storage (based on the assumption that $N$ is small - less than 256). In the preprocessing stage we introduce an array pos of byte such that, for each $i \in 1 . . N(n+1), \operatorname{pos}[i]=k$ iff $i$ is a position in $s_{k}$, while otherwise $\operatorname{pos}[i]=0(s[i]$ is a sentinel). Thus for every $i, \operatorname{pos}[i] \in 0 . . N$. Using divpts, pos can easily be computed in $\Theta(N n)$ time. Then, in order to determine, for each position $h$ in $s a$ which substring $s_{k}$ the position $s a[h]$ occurs in, it is necessary only to compute $k \leftarrow \operatorname{pos}[s a[h]]$. This $O(1)$ computation replaces BinarySearch in MultiRep-1, reducing processing time to $O(N n+\alpha)$, thus asymptotically optimal.

\subsection{Restricted Gaps (MultiRepG)}

In this section, we introduce the algorithm MultiRepG, for which the input is a maximal multirepeat $(p ; i, j)$ of $s$ satisfying constraints $D=\left(p_{\min }, q, m_{\min }\right)$ and the output consists of the elements of $(p ; i, j)$ that satisfy the gap constraints $d$ in at least $q$ substrings $s_{k}$ of $s$.

In order to satisfy constraints $d$ in addition to those specified by $D$, we need to introduce a bit vector $l o c=\operatorname{loc}[1 . . N(n+1)]$. In a single preprocessing stage every position in loc is set FALSE in time $O(N n / w)$, where $w$ is the computer word length, and the precondition $l o c[h]=$ FALSE for all $h$ is maintained thereafter. Then for each maximal repeat $(p ; i, j)$ of period $p \geq p_{\min }$, the positions $\operatorname{loc}[s a[h]], h=i, i+1, \ldots, j$, are set TRUE, so that a left-to-right scan of $l o c$ will yield in increasing order the positions of the repeating substrings in $s$. Such a scan is shown in Figure 3, used to determine which of the substrings $s_{k}$ in $s$ satisfy the gap constraints. A Boolean array gapsok $=$ gapsok $[1 . . N]$ is used to record the values $k \in 1 . . N$ for which $s_{k}$ satisfies $d$ (see the corresponding array mok described earlier for MultiRep-1). Algorithm MultiRepG executes in two phases, a checking phase and an output phase.

In the checking phase, divpts is used to compute for each $s_{k}$ an array occ of candidate positions. The function check, described below, actually applies the constraints $d$ to occ - its total time usage over all invocations is $O(r)$, where $r=j-i+1<N n$; also, the positions inspected in divpts and gapsok for each repeat are at most $N$. Thus for each candidate repeat, the time required to evaluate the constraints $d$ is $O(N n)$. For $R$ such repeats, the overall time requirement of the checking phase is therefore $O(R N n)$. We note that since $\alpha \leq R N n$ (the total number $\alpha$ of occurrences of repeats cannot exceed the number $R$ of repeats times the overall string length $N n$ ), therefore $O(R N n)$ in fact represents the total time required both for MultiRep- 2 and the checking phase. For cases that arise in practice, a corresponding statement holds also for MultiRep-1.

In the output phase, there is no action if less than $q$ substrings of $s$ contain repeats satisfying the constraints $d$. Otherwise, occ is recomputed for each $s_{k}$ that satisfies $d$ and the repeat is then output. For the strings and gap constraints of Example 2, described above, the output of the algorithm given in Figure 3 would be $(p, k, o c c)=(3,1 ; 1,5,8,14)$ and $(3,2 ; 4,9,17)$. The overall time requirement of the output phase is again $O(R N n)$.

The Boolean function check, shown in Figure 4, slides a window of width $m_{\min }$ over the $m \geq$ $m_{\min }$ entries in $o c c$, corresponding to the substring $s_{k}$, shifting right by one position at each step. For each window, check determines whether its entries satisfy the constraints $d$; if so, check returns TRUE, causing the $m$ repeating substrings of occ that occur in $s_{k}$ to be output. If no window of $o c c$ satisfies 


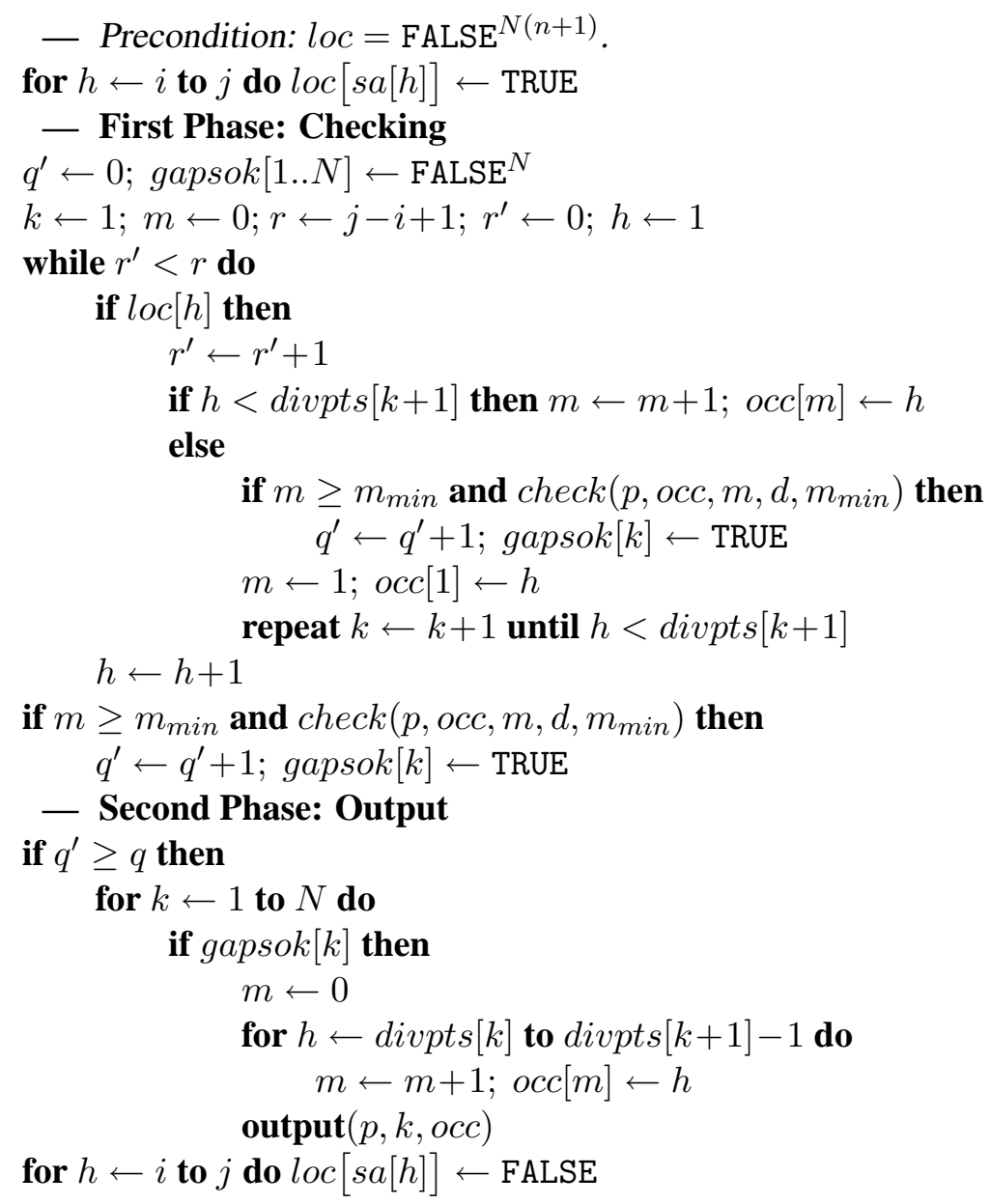

Figure 3. Algorithm MultiRepG: for each substring $s_{k}$ of $s$, if $o c c$ contains a sequence of length $\mu=m_{\min }$ that satisfies (1), then output $o c c$

$d$, check returns FALSE. The constraints $d$ are accessed as a two-dimensional array $d\left[1 . . m_{m i n}-1,1 . .2\right]$. The outer while loop of check is executed $\left(m-m_{\min }+1\right)$ times in the worst case, and the inner while loop is executed at most $m_{\min }$ times; thus the execution time of check at each invocation is $O\left(m_{\min }\left(m-m_{\min }+1\right)\right)=O(m)$. Here we assume that the specified input value $m_{\min }$ is constant over the execution of the algorithm. Over all invocations, therefore, the execution time of check is $O(r)$.

We note that the corresponding algorithm described in [1] requires that the differences between the maximum and minimum gaps specified in (1) should all be bounded by a small constant $c$. The methodology described here requires no such bound, and its effectiveness does not depend on such differences. Note also that MultiRepG can easily be modified, with the same asymptotic complexity and usage of space, to output only those ranges of occ that satisfy $d$, omitting those entries that do not.

The additional storage required for MultiRepG consists of the $4 N n / w$ bytes for loc plus up to $4 n$ bytes for the integer array occ, a total of $4 n(N / w+1)$. For $w=32$, this amounts to $n(N / 8+4)$, perhaps as much as an additional $N n$ bytes on top of the $9 N n$ used by MultiRep-1. 


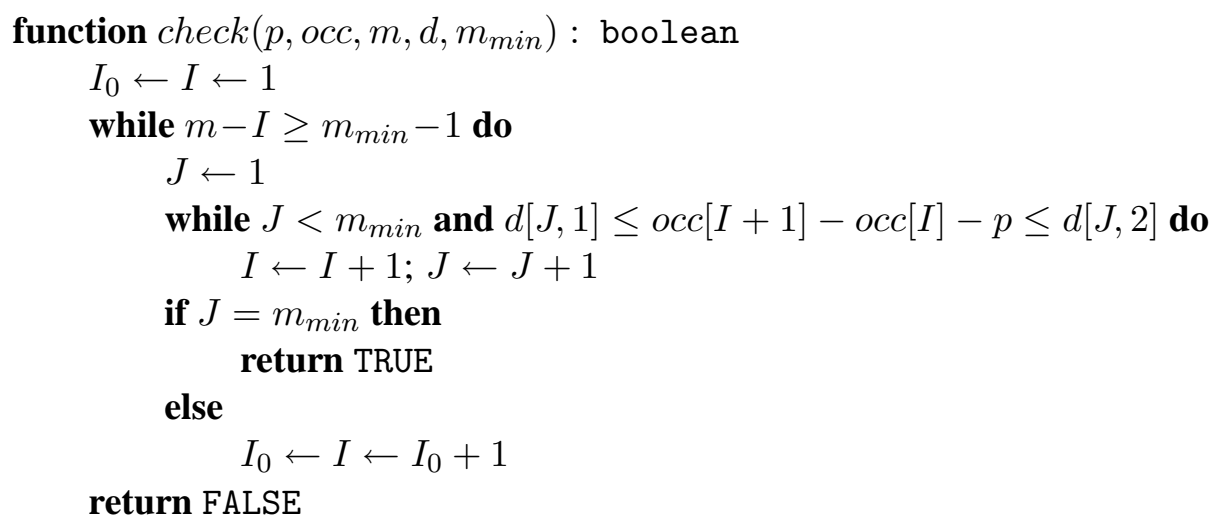

Figure 4. Function check: given an array occ of $m$ occurrences of a repeating substring in $s_{k}$, determine whether occ contains a subarray of length $\mu=m_{\min }$ that satisfies the constraints $d$

Table 1 compares the algorithms described here with those proposed in [1]. Note that even though

\begin{tabular}{l|c|c|c}
\hline Problem & Algorithm & Time & Space \\
\hline MultiRep & {$[1]$} & $O\left(\sigma N^{2} n+\alpha\right)$ & linear but large \\
& MultiRep-1 & $O\left(N n+\alpha \log _{2} N\right)$ & $9 N n$ bytes \\
& MultiRep-2 & $O(N n+\alpha)$ & $10 N n$ bytes \\
\hline MultiRepG & {$[1]$} & $\left.O\left(\left(c^{2}+\sigma^{2}\right) m N^{2} n \log (N n)\right)+\alpha\right)$ & $O\left(c^{2} N n m\right)$ \\
& MultiRepG & $O(R N n)$ & $10 N n$ bytes \\
\hline
\end{tabular}

Table 1. Comparison of Algorithms.

the suffix tree storage is linear, the large amount of information in each edge and node makes the suffix tree very expensive, consuming about ten to twenty times the memory size of the input text in good implementations. In [1], the algorithm MultiRep uses suffix trees, one for each string in the set plus a "generalized" suffix tree for all of them, therefore the memory usage would be very large.

\section{Discussion}

We have formulated two problems related to multirepeats in sets of strings with various restrictions and presented efficient algorithms with lower time complexity and less memory consumption compared to previously proposed algorithms. We remark that if in Algorithm MultiRepG we set the min and max constraints on gaps equal to zero, we can find all tandem repeats (repetitions) in arbitrary subsets of $S$. Future work includes the detection of degenerate (approximate) multirepeats and weighted multirepeats. 


\section{References}

[1] A. Bakalis, Costas S. Iliopoulos, Christos Makris, Spyros Sioutas, Evangelos Theodoridis, Athanasios K. Tsakalidis, Kostas Tsichlas: Locating maximal multirepeats in multiple strings under various constraints, The Computer Journal 50-2, 2007, 178-185.

[2] Michael Burrows, David J. Wheeler: A Block-Sorting Lossless Data Compression Algorithm, Technical Report 124, Digital Equipment Corporation, 1994.

[3] Johannes Fischer, Volker Heun, Stefan Kramer: Optimal string mining under frequency constraints, Proc. 10th European Conf. on Principles and Practice of Knowledge Discovery in Databases, LNCS 4213, Springer-Verlag, 2006, 139-150.

[4] Dan Gusfield: Algorithms on Strings, Trees and Sequences, Cambridge University Press, 1997.

[5] Juha Kärkkäinen, Peter Sanders: Simple linear work suffix array construction, Proc. 30th Internat. Colloq. Automata, Languages \& Programming, LNCS 2971, Springer-Verlag, 2003, 943-955.

[6] Toru Kasai, Gunho Lee, Hiroki Arimura, Setsuo Arikawa, Kunsoo Park: Linear-time longest-common-prefix computation in suffix arrays and its applications, Proc. 12th Annual Symp. Combinatorial Pattern Matching, LNCS 2089, Springer-Verlag, 2001, 181-192.

[7] Pang Ko, Srinivas Aluru: Space efficient linear time construction of suffix arrays, Proc. 14th Annual Symp. Combinatorial Pattern Matching, R. Baeza-Yates, E. Chávez \& M. Crochemore (eds.), LNCS 2676, SpringerVerlag, 2003, 200-210.

[8] Sau Dan Lee, Luc De Raedt: An efficient algorithm for mining string databases under constraints, Proc. KDID, LNCS 3377, Springer-Verlag, 2005, 108-129.

[9] Giovanni Manzini: Two space saving tricks for linear time LCP computation, Proc. 9th Scandinavian Workshop on Algorithm Theory, LNCS 3111, Springer-Verlag, 2004, 372-383.

[10] Simon J. Puglisi, W. F. Smyth, Munina Yusufu: Fast optimal algorithms for computing all the repeats in a string, Prague Stringology Conference, Jan Holub \& Jan Žd'árek (eds.), 2008, 161-169.

[11] Simon J. Puglisi, W. F. Smyth, Andrew Turpin: A taxonomy of suffix array construction algorithms, ACM Computing Surveys 39-2, Article 4, 2007.

[12] Bill Smyth: Computing Patterns in Strings, Pearson Addison-Wesley, 2003. 